Bisher konnten Kinder unter zwei Jahren nur mit monovalenten Konjugatimpfstoffen gegen C-Meningokokken geimpft werden, da herkömmliche polyvalente Polysaccharidimpfstoffe für diese Altersklasse nicht zugelassen sind. Ein breiterer Impfschutz ist laut Marshall aufgrund zunehmender Globalisierung und damit einhergehender Reisetätigkeit - auch von Eltern mit ihren Kindern - bedeutsam, zumal die globale Serogruppenverteilung großen regionalen Schwankungen unterworfen ist.

Im April 2012 wurde ein neuer 4-valenter Meningokokken-Konjugatimpfstoff zugelassen, der bereits Kinder ab dem vollendeten ersten Lebensjahr vor vier krankheitsauslösenden Serogruppen von Neisseria meningitidis schützen kann. Der neue
Konjugatimpfstoff MenACWY-TT (Nimenrix $^{\top M}$ ) enthält vier Schlüsselantigene der Bakterienkapsel (A, C, W-135, Y) die zur besseren Immunogenität an das Trägerprotein Tetanustoxoid (TT) konjugiert wurden. Studien zufolge konnte damit in jeder Altersgruppe ab dem vollendeten zwölften Lebensmonat bereits nach einer einmaligen Impfdosis eine gute Immunantwort und ein immunologisches Gedächtnis ausgebildet werden. Der neue Impfstoff wird in der Regel gut vertragen und kann gleichzeitig mit jedem anderen Kinderimpfstoff verabreicht werden.

Dr. Martina-Jasmin Utzt

"GSK Vaccines: Contributions to the prevention of bacterial meningitis" im Rahmen des 30. Kongresses der European Society of Paediatric Infectious Diseases (ESPID), Thessaloniki/Griechenland, 9. Mai 2012; Veranstalter: GSK

\section{Klinische Wirksamkeit von PC13} Seit dem Jahr 2006 besteht in Deutschland eine allgemeine Empfehlung der STIKO am Robert-Koch-Institut für die Pneumokokken-Konjugatimpfung. Sechs Jahre danach gibt es fast keine Fälle von invasiven Pneumokokkenerkrankungen (IPD) mehr, die durch in PCV 7 enthaltene Serotypen ausgelöst wurden. Zwei Jahre nach der Einführung von der 13-valenten PCV13 werden bereits Effekte der sechs neuen Serotypen bei Kindern unter zwei Jahren sichtbar, v. a. von Serotyp 19A. „Es ist sehr ermutigend zu sehen, dass bereits zweieinhalb Jahre nach Einführung von Prevenar13 ${ }^{\circ}$ in Deutschland solch ein beträchtlicher Rückgang der registrierten IPD-Fälle erreicht werden konnte", sagte Dr. Mark van der Linden vom Universitätsklinikum Aachen, wo seit 1997 am Nationalen Referenzzentrum für Streptokokken eine Serotypisierung von Fällen mit IPD stattfindet. „Diese Ergebnisse belegen den Nutzen der Imfung gegen IPD und verdeutlichen die Notwendigkeit einer breiten Serotypenabdeckung."

Nach Informationen von Pfizer

Viel versprechende Wirkung bei SJIA

Die systemische juvenile idiopathische Arthritis (SJIA) ist eine seltene, systemische, IL-1 $\beta$-vermittelte autoinflammatorische Erkrankung, charakterisiert durch täglich auftretende Fieberschübe, Hautausschlag, chronische Schmerzen und Arthritis. In einer Phase-III-Studie wurde nun die Wirkung von ACZ885 (Canakinumab), einem humanen monoklonalen Antikörper, der Interleukin 1 beta (IL-1 $\beta$ ) inhibiert, getestet: Bei $62 \%$ der mit ACZ885 behandelten SJIA-Patienten konnte am Ende der placebokontrollierten Phase ein inaktiver Krankheitsstatus belegt werden. Die Patienten waren symptomfrei - ohne aktive Arthritis, Fieber und rheumatoiden Hautausschlag - und wiesen normalisierte Werte von Entzündungsparametern wie Blutsenkungsgeschwindigkeit und CRP auf.

Dagegen war das nur bei $32 \%$ der Patienten der Fall, die zunächst ebenfalls ACZ885 erhalten hatten und dann randomisiert mit Placebo weiterbehandelt wurden. 33\% der mit ACZ885 behandelten SJIA-Patienten konnten die vorher benötigten Kortikosteroide vollständig absetzen.

Nach Informationen von Novartis 